\title{
Le rôle de l'Etat-Congrès et des acteurs extérieurs dans la montée du séparatisme sikh
}

Anne Vaugier

\section{(2) OpenEdition \\ 1 Journals}

\section{Édition électronique}

URL : http://journals.openedition.org/conflits/513

DOI : $10.4000 /$ conflits.513

ISSN : $1777-5345$

Éditeur :

CCLS - Centre d'études sur les conflits lilberté et sécurité, L'Harmattan

Édition imprimée

Date de publication : 15 octobre 1994

ISSN : 1157-996X

\section{Référence électronique}

Anne Vaugier, « Le rôle de l'Etat-Congrès et des acteurs extérieurs dans la montée du séparatisme sikh », Cultures \& Conflits [En ligne], 15-16 | automne-hiver 1994, mis en ligne le 15 mars 2006, consulté le 30 mars 2021. URL : http://journals.openedition.org/conflits/513 ; DOI : https://doi.org/10.4000/ conflits.513

Ce document a été généré automatiquement le 30 mars 2021.

Creative Commons License 


\title{
Le rôle de l'Etat-Congrès et des acteurs extérieurs dans la montée du séparatisme sikh
}

\author{
Anne Vaugier
}

1 Les Sikhs, adeptes du sikhisme, foi monothéiste, expression syncrétique de l'hindouisme et de l'islam, fondée sur les terres du Pendjab à la fin du quinzième siècle par Guru Nanak (1469-1539), ne représentent, avec quelques 17 millions de personnes, que $2 \%$ de la population indienne. Si la place des Sikhs dans la mosaïque plurireligieuse de l'Inde parait de ce fait moindre, (les hindous représentant $83 \%$ de la population, les musulmans $11,3 \%$, les chrétiens $2,4 \%$, les bouddhistes $0,7 \%$, les jains 0,5 $\%$ ), cette relative faiblesse numérique a toujours été compensée par la place prépondérante occupée par les Sikhs dans la vie politique et économique de l'Inde coloniale puis de l'Inde indépendante. Historiquement, le communautarisme sikh, dans ses manifestations culturelles, remonte à la fin du dix-neuvième siècle et plus précisément à 1870, date à laquelle furent créées les premières " Singh Sabhas ", " assemblées " ou " congrégations " sikhes à vocation religieuse et éducative, destinées à renforcer le sentiment de cohésion du groupe sikh face à un hindouisme prompt à absorber les minorités. En 1920, l'apparition d'une nouvelle force politique, l'Akali Dal, parti représentant les intérêts des Sikhs, fondé en vue d'enlever la gestion des " gurudwaras ", lieux de culte sikhs, aux prêtres hindous pour les restituer aux Sikhs, marqua une étape décisive dans le processus d'institutionnalisation du caractère séparé des Sikhs. Enfin, la politique préférentielle des Britanniques à l'égard des Sikhs, considérés comme une " race martiale ", jusqu'à la fin de l'Empire en 1947, ne fit que conforter le sentiment sikh d'appartenir à un " peuple élu " ${ }^{1}$. Au moment de la partition de 1947, les Sikhs eurent un sursaut identitaire, prenant trop tardivement conscience qu'un partage du pays suivant un clivage confessionnel, séparant hindous et musulmans, ne pourrait être évité et qu'ils en seraient les premières victimes. Ils devraient désormais trouver leur place dans une Inde laïque, mais peuplée à presque 85 \% d'hindous. Depuis 1947, les revendications d'intégration sikhes mettent à l'épreuve la capacité du système fédéral indien à intégrer une des communautés indiennes dans les 
milieux structurés. Dans ce contexte, le communautarisme sikh apparaît comme l'expression d'une double volonté: une première de nature endogène, celle d'une minorité religieuse de se souder et de se structurer face à la communauté hindoue majoritaire, à travers des institutions qui lui sont propres comme le Shiromani Gurudwara Prabandhak Committee (SGPC), organe de gestion des temples, ou l'Akali Dal, porte-parole des intérêts des Sikhs. Au lendemain de la partition, la puissance du communautarisme s'exprima ainsi dans un mouvement de renaissance linguistique et religieuse qui se poursuivit jusqu'au milieu des années soixantes, caractérisé par la lutte pour l'obtention d'un Etat pendjabiphone ou " Punjabi Suba " 2. D'autre part, ce communautarisme procède d'une volonté exogène, traduite par le détournement d'allégeances primordiales, au profit d'institutions et de partis qui sont l'expression d'un consensus national. Dans ce cadre, il nous appartiendra d'analyser comment le communautarisme sikh, enraciné dans l'histoire, a été entretenu voire manipulé par le Parti du Congrès, vecteur du mouvement nationaliste puis détenteur du monopole quasi-exclusif du pouvoir au Centre et dans de nombreux Etats de l'Union depuis 1947. Enfin, l'influence de la diaspora et le soutien de pays voisins demeurent des facteurs essentiels pour appréhender l'origine de la consolidation et des effets pervers d'un mouvement communautariste qui a conduit à l'une des crises majeures de l'Inde indépendante.

2 La constitution du communautarisme sikh après 1947 Les Sikhs pouvaient-ils être les bénéficiaires privilégiés du patronage de l'Etat, soutenir le parti du Congrès et conserver leur identité en tant que Sikhs? De telles questions ont toujours préoccupé les membres du " Panth " - terme consacré désignant la communauté sikhe - et ses dirigeants depuis le début des années cinquante. Au départ, l'intégration des Sikhs dans l'Union indienne fut certes favorisée par l'attitude ambiguë du parti du Congrès qui se portait garant des intérêts sikhs à l'échelle régionale. Ainsi, l'absence d'opposition radicale des membres du parti Akali au parti du Congrès fut sans doute influencée par le laxisme avec lequel ce dernier orchestra la politique agraire relative aux plafonds agraires au Pendjab. Dans les années cinquantes, la lenteur de mise en oeuvre des réformes agraires contribua en effet très largement à encourager les Jats, caste très bien représentée parmi les propriétaires terriens, à se ranger aux côtés du parti du Congrès en mettant opportunément en sourdine toute revendication " régionale ". Par la suite, le parti du Congrès, loin de s'opposer aux intérêts sikhs, joua la carte de l'attentisme, tempérant les exigences des Sikhs et espérant ainsi calmer leurs revendications d'ordre politique, social et culturel. Plus que l'expression d'un choix volontaire du Congrès de composer avec les minorités, il faut voir dans cette attitude la marque d'une première faiblesse d'un parti aux ambitions nationales mais aux assises fragiles sur le plan régional. En effet, en dépit de " banques de votes ", c'est-à-dire des votes captifs des " hindous urbains ", des " basses castes " et des " Sikhs ruraux ", le Congrès ne put qu'à deux reprises gouverner l'Etat du Pendjab, en 1972 et 1980. Cette extrême fragilité politique justifie la politique de la main tendue choisie par le Congrès, qui se traduisit notamment par la mise sur pied de la "States Reorganization Commission " en 1953, visant à retracer les frontières de plusieurs Etats et ainsi satisfaire aux exigences des communautés revendiquant un redécoupage territorial obéissant au critère de l'homogénéité linguistique. Par ces concessions faites au lendemain de l'indépendance, l'Etat indien marquait sa tolérance à l'égard des particularismes existants, et entendait prouver qu'il ne considérait pas la présence de communautarismes comme une menace à l'intégration nationale. Master Tara Singh, 
leader de l'Akali Dal jusqu'en 1965, avait revendiqué, dès 1948, un redécoupage de l'Etat, séparant les régions hindiphones des régions pendjabiphones, sans cacher son objectif ultime de création d'un Etat à majorité sikhe ${ }^{3}$. La campagne menée parallèlement au Pendjab par le magnat du plus grand groupe de presse hindoue, Lala Jagat Narain, exhortant les Pendjabis hindous à déclarer l'hindi comme étant leur langue maternelle, avait parachevé la polarisation des communautés hindoues et sikhes sur la question d'un Punjabi suba (Province [parlant] Pendjabi). Aussi, lorsque Tara Singh réitéra sa demande devant la Commission de Réorganisation des Etats, celle-ci la rejeta en 1955, la qualifiant de communautariste, Jawaharlal Nehru adopta la même position. En 1965, Sant Fateh Singh, prêcheur Jat-sikh, succédait à Tara Singh à la tête de l'Akali Dal et dotait sa requête d'une nouvelle légitimité, en demandant un Etat linguistique séparé pour ceux qui parlaient pendjabi et utilisaient l'alphabet gurumkhi, tandis qu'il reléguait les préoccupations d'essence religieuse au second plan. L'épisode de la lutte pour la Punjabi Suba fut révélateur de l'importance du contexte dans lequel s'inscrivent les revendications particularistes. En 1966, le changement de direction au Centre, Indira Gandhi succédant à Lal Bahadur Shastri, et les bons et loyaux services rendus par les soldats sikhs lors du conflit indo-pakistanais de 1965, permirent à Sant Fateh d'obtenir gain de cause, le patriotisme sikh ne pouvant plus être mis en doute. Le Pendjab fut à nouveau divisé, les régions essentiellement pendjabiphones, attribuées au Pendjab, les régions hindiphones à l'Haryana et les régions montagneuses à l'Himachal Pradesh. Après cette division, $5 \%$ des Sikhs demeurèrent en Haryana et $30 \%$ d'hindous dans le nouvel Etat du Pendjab. L'obtention d'un Etat majoritairement sikh loin d'être une fin en soi, fut le point de départ de nouvelles revendications du Panth ${ }^{4}$. Ayant largement bénéficié de la Révolution Verte à partir de la fin des années 1960, les Sikhs constituent la seule minorité privilégiée et prospère d'Inde et, de ce fait, apparaissent avant tout soucieux de consolider leurs assises politiques et économiques. L'un des principaux griefs de la communauté sikhe à l'encontre du parti du Congrès tenait à ce que le vivier de ses partisans se trouvât au sein des membres des ex-intouchables désignés depuis 1947 comme castes répertoriées (scheduled castes) et des " banias " (membres des castes marchandes) hindous. Ces deux catégories entretenaient des rapports tendus avec les Sikhs car ces derniers, agriculteurs en majorité, utilisaient les premiers comme ouvriers agricoles et dépendaient des seconds pour écouler leur production. L'essoufflement économique annoncé par les prévisionnistes en raison d'un développement essentiellement fondé sur le secteur primaire sans perspective d'ouverture du secteur secondaire, fit craindre à la communauté sikhe et particulièrement aux jeunes générations de Sikhs Jat qu'un gouvernement congressiste ne soit pas en mesure de mettre en oeuvre une politique de croissance économique. Tandis que le parti Akali se projetait en défenseur des intérêts de la classe des Jats Sikhs, le parti du Congrès s'employa à partir de 1972 à apparaître comme le protecteur des intérêts religieux sikhs, un rôle traditionnellement assumé par l'Akali Dal conjointement avec le SGPC.

3 L'Etat-Congrès et la manipulation de l'identité sikhe Dès le début des années soixantedix, le parti du Congrès, représentant l'Etat, entreprit de manipuler le communautarisme sikh afin de contrer l'influence des Sikhs modérés qui n'avait cessé de s'affirmer depuis l'obtention d'un Etat à majorité sikhe ${ }^{5}$. Le mandat de Giani Zail Singh, promu chef du gouvernement de l'Etat du Pendjab en 1972, prouva que le parti du Congrès n'hésitait pas à utiliser l'appareil d'Etat pour étendre son influence, non seulement dans le domaine politique local mais aussi dans la sphère religieuse. Cette 
opération de propagande contrastait avec l'image de parti laïque, projetée par le parti du Congrès à l'échelle nationale ${ }^{6}$. Champion de l'instrumentalisation du religieux, Giani Zail Singh, ancien membre du parti Akali Dal passé dans les rangs du parti du Congrès, entreprit de construire une route reliant les gurudwaras et alla jusqu'à rebaptiser rues, villes et institutions du nom des gourous du panthéon sikh. Le parti régional, l'Akali Dal, porte-parole des intérêts sikhs, se voyait battu sur son propre terrain. Sa revanche politique ne fut prise qu'au moment de la proclamation de l'état d'urgence par Indira Gandhi en 1975, où il fut le seul parti à manifester contre cette offense à la démocratie. A la même période, le parti Akali formulait, pour contrer l'offensive du Parti du Congrès, un programme à teneur politique et économique, nettement favorable aux Sikhs : la Résolution d'Anandpur Sahib. Dans l'une des versions anglaises du document figure clairement énoncé l'objectif fondamental de l'Akali Dal : " The Shiromani Akali Dal has been guiding the sikhs according to the exigencies of the situation for their rights and for a respectable status in the set-up of the country. Its objective has been the exaltation of the Sith Panth and for achievement of this lofty ideal, the Shiromani has been adopting different and various means " ${ }^{7}$. Au titre des idées maîtresses de ce document, considéré comme la charte des revendications sikhes, figurent la concession d'un statut d'autonomie à l'Etat du Pendjab, l'octroi de Chandigarh, une capitale que le Pendjab partage avec l'Haryana, au seul Pendjab et des régions pendjabiphones adjacentes au Pendjab, et l'attribution du statut de seconde langue au Pendjabi dans les Etats voisins du Pendjab. Ce document qui scellait officiellement le clivage entre hindous et sikhs, fait, depuis sa promulgation en 1978, l'objet d'une controverse non résolue. S'agit-il d'une liste de revendications légitimes dans le cadre d'une décentralisation plus poussée au niveau des Etats ou de la charte d'un nationalisme sikh ? Au sein du parti Akali, ces deux interprétations sont également représentées. Les membres de la faction Longowal, la faction modérée des Akalis, se sont toujours opposés au slogan d'un Khalistan (Etat sikh indépendant) qui apparut peu après et se sont déclarés en faveur de l'unité et de l'intégrité du pays sans démentir toutefois leur adhésion de principe à la résolution d'Anandpur Sahib, en en faisant même la condition de la reprise du dialogue avec le gouvernement central. Si de par sa teneur, la résolution s'insère dans le cadre d'une réflexion plus large sur la répartition des pouvoirs au sein du système fédéral indien, l'identité religieuse et politique sikhe s'affirme sans ambiguïté dans les clauses revendiquant une garantie des droits fondamentaux des minorités religieuses et linguistiques pour répondre aux exigences d'une tradition démocratique, dont le Parti du Congrès s'était porté garant. En 1977, le parti Akali constitua avec le parti Janata un gouvernement de coalition qui allait durer deux ans, de 1977 à 1979. Durant cette phase, les dirigeants akalis s'efforcèrent de minimiser leur utilisation de symboles religieux en raison de leur alliance avec un parti laïque, le parti Janata. Cette phase constitue une parenthèse politique, répondant à des aspirations intercommunautaires avant le basculement dans le communautarisme au début des années quatre-vingt. Les valeurs culturelles et sociales, et les aspirations communes des Pendjabis hindous et sikhs, prenaient le pas sur les particularismes religieux. Ce gouvernement sous l'égide d'un sikh jat, Prakash Singh Badal, jouissait de l'appui des deux groupes économiquement dominants de la région, les Sikhs jats des zones rurales et les hindous des classes industrielles et commerçantes des villes, soit la catégorie de la population pendjabie bénéficiaire de la révolution verte, et partageant le même souci de maintenir la stabilité politique régionale. A la fin des années 1970, paradoxalement, l'exploitation du sentiment d'appartenance communautaire qui 
déboucha rapidement sur une dérive communautariste, fut le fait non d'un parti fortement en faveur des valeurs et idéaux propres au sikhisme, comme l'Akali Dal, mais d'un parti qui s'affichait depuis l'indépendance comme le défenseur d'une Inde laïque, dissociant radicalement le religieux du politique, le parti du Congrès. Aussitôt après sa défaite de 1977, le Parti du Congrès joua en effet la carte de la surenchère religieuse pour affaiblir son principal rival politique au Pendjab, l'Akali Dal, et promut un prêcheur sikh du nom de Jarnail Singh Bhindranwale, à la tête d'un mouvement qui, de " revivaliste ", allait rapidement dégénérer en mouvement fondamentaliste puis terroriste et échapper à tout contrôle. C'est à des dirigeants sikhs du parti du Congrès, nommément Giani Zail Singh et Darbara Singh, cédant en cela aux injonctions de Sanjay Gandhi, le fils cadet d'Indira Gandhi, que l'on attribue l'idée d'utiliser un Sant sikh pour faire pièce au parti Akali. Bhindranwale apparaissait comme un élu de premier choix. A la tête d'une formation puriste, le Damdami Taksal, ce dernier n'avait jamais caché son penchant pour le fondamentalisme et sa foi en l'action violente, mais le parti du Congrès lui avait accordé son soutien sans réserves. En 1978, ce dernier déclencha un mouvement d'action violente. Arguant d'une dégradation de l'orthodoxie au sein du Panth Sikh, il prit pour cible le mouvement Nirankari, secte hétérodoxe du sikhisme, dont il assassina le chef de file, Baba Gurcharan Singh en 1980. Le mouvement extrémiste se poursuivit dans cette mouvance, avec le meurtre en 1981 de Lala Jagat Narain, patron du groupe de presse Hind Samachar. Après sa campagne de presse contre la création d'une Punjabi Suba, cette figure du mouvement de l'Arya Samaj, mouvement revivaliste hindou créé en 1875 , ne s'était jamais départi de ses prises de position ouvertement pro-hindoues. Paradoxalement, loin de réagir à ces attentats, le gouvernement central s'employa au contraire activement, de 1979 à 1983, à consolider les assises de Jarnail Singh Bhindranwale. L'alliance Congrès/Bhindranwale fut rendue publique lors de la campagne électorale pour les élections générales de 1980, quand Bhindranwale fut aperçu partageant le dais d'Indira Gandhi dans la localité de Gurdaspur. L'échec du gouvernement dans la condamnation des coupables des attentats marquait la première faille de cette contre-stratégie ethnique de l'Etat, visant à galvaniser les membres du Panth, par l'emprise d'un leader charismatique et permettait d'anticiper les débordements à venir. La première riposte à cette stratégie s'opéra en 1982, lorsqu'une faction modérée du parti Akali, sous l'égide de Sant Harchand Singh Longowal, lança une série de " morchas " ou manifestations de résistance passive exprimant ainsi son désaccord vis-à-vis de la politique du gouvernement en place. Les partisans de Bhindranwale se joignirent à ces manifestations, acte qui fut perçu par l'opinion publique comme le sceau de l'alliance des modérés et des extrémistes. La stratégie de résistance passive, lancée par l'Akali Dal, " Nahar Roko ", " Rasta Roko ", " Kam Roko ",à savoir cesser la construction du canal, des routes, toute activité, culmina en une " Dharm Yuddh ", ou guerre sainte, laquelle allait se prolonger jusqu'en 1985. L'identité communautaire sikhe, reformulée par Bhindranwale devint exclusive et agressive. L'emprise du mouvement peut s'expliquer par les conséquences induites par la prospérité économique dans le monde paysan, sur les jeunes sikhs qui anticipaient un essoufflement de la croissance. Bhindranwale représentait le besoin d'une identité sikhe clairement redéfinie et le frein nécessaire à la perte de traditions induite par la modernisation. La All India Sikh Students Federation (AISSF) constitua-t-elle une pièce maîtresse d'un mouvement dirigé par Amrik Singh mais dont Bhindranwale fut le leader charismatique? Des doutes demeurèrent toujours quant à l'aura de sainteté dont jouissait ce dernier. Ses 
disciples ne surent jamais s'il agissait pour le compte du Panth ou pour celui de certains leaders congressistes. Le succès du mouvement lancé par Bhindranwale refléta la capacité de l'Etat-Congrès à manipuler et exploiter les mouvements communautaristes. Après 1985, les modérés dans l'Etat du Pendjab allaient être radicalement marginalisés. L'échec d'une ébauche de conciliation en 1985, l'accord Rajiv Gandhi - Sant Longowal, imputé au refus du parti du Congrès de veiller à son application, provoqua une déroute réelle des modérés qui avaient repris les rênes du pouvoir en septembre de la même année. Mais le mouvement communautariste sikh, puis ouvertement séparatiste, n'aurait pu survivre sans l'important soutien dont il bénéficia à l'étranger.

Le rôle de la diaspora et des voisins de l'Inde C'est hors d'Inde que le communautarisme sikh a puisé son idéologie et son soutien logistique pendant une décennie. L'internationalisation de l'action de contestation s'opéra principalement à travers les organisations séparatistes sikhes fondées au Royaume-Uni, en Allemagne, au Canada et aux Etats-Unis au début des années quatre-vingt. Que les membres de la communauté sikhe constituent la majeure partie des indiens expatriés et qu'ils aient conservé des liens économiques et affectifs avec la mère-patrie n'a jamais été contesté. L'immigration sikhe dans le monde anglo-saxon est ancienne. Elle s'est faite en trois vagues successives. Jusqu'à la fin de la Seconde Guerre Mondiale, les Sikhs du RoyaumeUni se comptaient par milliers. Cette communauté était composée essentiellement d'étudiants, de membres de professions libérales. La seconde vague d'immigration fut provoquée par la partition du sous-continent en 1947. Les Sikhs en provenance du Pakistan affluèrent en Angleterre, suivis de ceux d'Afrique Orientale. La dernière vague d'immigration fut générée par l'Opération Blue Star (Juin 1984) et par l'explosion de violence manifestée à l'encontre des Sikhs après l'assassinat d'Indira Gandhi par ses gardes du corps sikhs. Jusqu'en 1984, les activités des communautés sikhes à l'étranger se résumaient à la gestion des gurudwaras de leurs localités. Après l'assaut du Temple d'Or par l'armée indienne, les nouveaux arrivants plus orthodoxes et politiquement plus motivés que les immigrés des premières générations prirent le contrôle des gurudwaras et transformèrent ces lieux de culte en forum de propagande antiindienne. Un nombre important d'organisations vit le jour, propageant l'idée d'un Khalistan et apportant leur soutien aux réseaux terroristes en Inde. L'idée d'un Khalistan fut elle-même celle d'un homme de la diaspora. Le concept d'Etat sikh fut élaboré par un ancien étudiant d'Oxford, Kapur Singh (né en 1909), membre de l'Indian Civil Service, prestigieux corps d'administration indien, émigré en Grande-Bretagne. C'est dans les années soixante que se développa l'idée de créer l'Etat du Khalistan, entité théocratique, " Pays des Purs ". Parmi les principales organisations sikhes, piliers du mouvement communautaire, le National Council of Khalistan, le Dal Khalsa, le Babbar Khalsa et l'Akhand Kirthani Jatha méritent une mention toute particulière. Le National Council of Khalistan avait ouvert sous l'égide de Jasjit Singh Chauhan des antennes au Royaume-Uni, en Allemagne, au Canada et aux Etats-Unis. Les activités du Dal Khalsa étaient quant à elles principalement lancées aux Etats-Unis et en Allemagne, tandis que le Babbar Khalsa agissait principalement depuis Vancouver. L'Akhand Kirthani Jatha opérait au Royaume-Uni et au Canada ${ }^{8}$. De toutes ces formations, c'est au National Council of Khalistan que l'on doit la création du slogan Khalistan, " pays des purs ", nouvelle entité territoriale aux consonances familières, prononcé pour la première fois à Londres en septembre 1971. Une décennie plus tard, le 12 avril 1980, Jasjit Singh Chauhan annonçait la formation du Conseil National du Khalistan et eut recours à de nombreux artifices pour asseoir les assises de la République du Khalistan 
proclamée par un gouvernement en exil sous son égide. L'émission de passeports, de timbres, de monnaie au sceau khalistani, parachevèrent l'officialisation du mouvement à l'étranger. Ces activités jugées anti-indiennes entraînèrent la confiscation du passeport indien de Chauhan en 1981 et des poursuites à son encontre pour activités séditieuses et encouragement à la haine entre les communautés. Bravant les mises en garde du gouvernement indien, Chauhan, durcit ses positions, anti-indiennes. Il brûla publiquement le drapeau indien, appela en octobre 1982, les leaders akalis à la désobéissance civile et à la non-coopération avec le gouvernement élu par la voie démocratique au Pendjab. Il alla jusqu'à revendiquer la genèse d'une force de 10000 hommes susceptible de s'engager dans une guérilla contre les troupes indiennes. En mai 1983, il se targuait d'avoir le soutien des Etats-Unis et de pouvoir ainsi atteindre ses objectifs en quatre ans. Il lança un appel pour mettre fin aux exportations de céréales à l'Inde. En juillet 1983, il conseillait à Sant Harchand Singh Longowal et à Bhindranwale de constituer rapidement un nouveau gouvernement sous peine sinon de voir le NCK s'en charger. Le Conseil fit circuler une résolution indiquant la volonté de création d'un foyer pour les Sikhs et la revendication d'une voix à l'Assemblée Générale des NationsUnies ${ }^{9}$. Ganga Singh Dhillon constitue une autre figure de proue du mouvement séparatiste de la diaspora. Citoyen américain d'origine indienne, il est président de la Nankana Sahib Foundation à Washington. Il rendit publique l'existence de liens entre les militants du JKLF (Jammu and Kashmir Liberation Front) et le NCK (National Council of Khalistan). Dès 1981, Chauhan revendiquait le soutien du parti Jamaat-e-Islami dans la partie du Cachemire sous contrôle pakistanais. Il parcourut à la même époque le pays en proclamant l'état de " nation séparée " (qaum) des Sikhs. L'entretien de liens avec des sénateurs américains, ainsi qu'avec des membres de la Haute Administration au Pakistan, fut capital pour la diffusion et le gain en crédibilité du mouvement ${ }^{10}$. Enfin, une autre organisation de premier plan, le Dal Khalsa, vit le jour dans la foulée de l'adoption de la résolution d'Anandpur Sahib, le 13 avril 1978, avec pour objet la création d'un Etat sikh indépendant et souverain. Contrairement aux deux précédentes, cette formation ne déguisa jamais son recours à l'usage de la force pour parvenir à ses fins, comme en témoigne le détournement d'un appareil d'Indian Airlines, le 29 septembre 1981. Interdite en Inde en mai 1982, au titre du " Unlawful Activities Prevention Act ", l'organisation poursuivit son activité depuis le Royaume Uni et l'Allemagne où elle ouvrit des branches en 1983. Au Pendjan, l'exercice de pressions pesantes sur les factions akalies modérées se fit ouvertement après l'annonce de la décision, prise conjointement par le Dal Khalsa et le Babbar Khalsa depuis Vancouver, de menacer les factions akalies modérées de subir un sort similaire à celui des Nirankaris en 1978. Ces mêmes organisations revendiquèrent l'assassinat du magnat de la presse hindoue Lala Jagat Narain, et de Shri Atwal directeur des forces de police du Pendjab, au début des années quatre-vingts. Le Dal Khalsa depuis l'Angleterre lançait explicitement un appel à la lutte armée au Pendjab et menaçait de mort " les traitres à la communauté ". Depuis Vancouver, à la même période, le " Consul-Général du Khalistan ", se targuait de l'appui du Pakistan, qui accorderait, après la constitution d'un Khalistan, à Nankana Sahib, lieu de pèlerinage sikh situé en territoire pakistanais, un statut similaire à celui du Vatican, L'Akhand Kirthai Jatha se démarqua des autres par sa vocation essentiellement religieuse, mais étendit son soutien aux autres organisations politiques, extrémistes sikhes, en particulier au Babbar Khalsa. Aussi, le mouvement séparatiste pour la création d'un Khalistan n'aurait-il pas connu un tel essor dans les années quatre-vingts sans l'incitation et l'inspiration reçue de la 
diaspora sikhe au Royaume-Uni, au Canada et aux Etats-Unis. L'Etat voisin du Pakistan entretint lui aussi le réseau militant en fournissant camps d'entraînement et soutiens logistiques aux militants khalistanis sur son territoire. Depuis le début des années quatre-vingts, de nombreux camps ont été identifiés par les services spéciaux indiens à Kothatial, Niazbeg, Sargodha, Attock, Gujranwala, Sobha Singh, Rawalpindi, Nankana Sahib, Rahimyar Khan. Les objectifs stratégiques et politiques du Pakistan étaient selon New Delhi, d'encourager les divisions hindous-sikhs afin d'établir une ceinture sikhe pro-pakistanaise, très importante comme base de renseignements sur les mouvements de troupes en cas de guerre, et d'essayer de détruire la cohésion politique et ethnique des Sikhs avec le reste du pays sans soutenir réellement l'idée de l'établissement d'un Etat théocratique à sa frontière. L'internationalisation de la question sikhe compta sans doute pour beaucoup dans la décision du Premier ministre Indira Gandhi de mettre radicalement fin au mouvement qu'elle avait généré et qui échappait désormais à tout contrôle. Le 2 juin 1984, Indira Gandhi annonçait à la Nation indienne l'action qu'elle était prête à entreprendre contre Bhindranwale et ses partisans, réfugiés dans le sanctuaire des Sikhs, le Temple d'Or d'Amritsar, transformé en arsenal et quartier général des terroristes. Accusant les représentants akalis, d'émettre sans cesse de nouvelles revendications et de fomenter campagne d'agitation sur campagne d'agitation, Indira Gandhi déclarait: " la direction de l'organisation semble avoir été saisie par un groupe de fanatiques et de terroristes dont les instruments pour atteindre leurs visées ne sont que meurtre, poudre et pillage " ${ }^{11}$. A l'absence persistante d'une politique étatique clairement définie à l'égard des minorités succéda l'émergence d'un appareil d'Etat répressif conçu pour répondre à toute attaque. L'assaut de l'armée fédérale et la destruction de l'Akal Takht-trône des Immortels, symbole du pouvoir spirituel et temporel du sikhisme fut une erreur politique considérable. Dans la foulée, l'opération " Woodrose ", fouille systématique des demeures des familles sikhes du Pendjab, donnant lieu souvent à des interrogatoires, voire même à des tortures, parachevèrent ce processus d'aliénation de la communauté sikhe. Les conséquences sociales, religieuses et politiques de l'opération Blue Star et des pogroms anti-sikhs de Delhi en novembre 1984 (qui firent plus de 2000 victimes et 10000 sans-abri), après l'assassinat du Premier Ministre, consacrèrent l'enlisement politique. Jusqu'à la disparition d'Indira Gandhi, les moindres revendications politiques émises par les minorités religieuses, furent perçues et projetées comme des menaces à l'unité et à l'intégrité nationale et violemment réprimées ${ }^{12}$. Fort du raz de marée électoral qui le porta au pouvoir en novembre 1984, Rajiv Gandhi, succédant à sa mère, opta pour une politique de main tendue et relança les négociations avec la faction modérée akalie. L'accord Rajiv Gandhi-Harchand Longowal, symbolisait le 24 juillet 1985, la victoire des forces en faveur de l'intégration nationale. Dans la foulée, les élections à l'assemblée régionale de septembre 1985 portèrent au pouvoir avec une imposante majorité de 73 sur 117 sièges, un gouvernement akali sous l'égide d'un Jat-sikh, Surjit Singh Barnala. Ce triomphe électoral permettait d'amorcer un processus de normalisation. Cependant, les défections dans les rangs Akali, et les promesses non tenues du parti du Congrès (Transfert de Chandigarh au Pendjab originellement prévu pour le 26 janvier 1986) ruinèrent ces espoirs. En sabotant l'accord Rajiv-Longowal, le gouvernement laissait le champ libre aux extrémistes revendiquant le leadership du Panth. Le 26 janvier 1986, la Constitution d'un Comité Panthique par une assemblée de la Khalsa redéclenchait la spirale de violence ${ }^{13}$. La répression policière massive de l'Etat, sous l'égide de Julio Ribeiro, qui opta pour une lutte anti-terroriste à tout crin, eut des conséquences 
dramatiques. De source officielle, le nombre des attentats passa de 1246 à 3047 entre 1987 et $1988^{14}$. Au début des années quatre-vingt dix, les terroristes profitèrent du vide politique dans l'Etat placé sous régime d'administration directe depuis mai 1987, en imposant à la population un code de conduite draconien. Loin de faire appel à un processus d'identification spontanée, à des références culturelles anciennes, ils tentèrent de les imposer par la force. Les directives des militants portèrent d'abord sur l'interdiction de consommer alcool et tabac, ainsi que sur la prescription d'un végétarisme strict, obéissant en cela à la lettre aux préceptes du sikhisme. Par la suite, ils imposèrent un code vestimentaire dans les écoles tandis que le Pendjabi y était imposé comme langue d'instruction. L'enjeu de la langue fut remis à l'ordre du jour et l'usage de l'hindi proscrit sur les ondes de la radio officielle, All India Radio ${ }^{15}$. Imposé par la force et non fruit d'un choix de la communauté dans son ensemble, ce retour aux sources et ces allégeances forcées n'ont été qu'éphémères. Le bref intermède que constitua le gouvernement de front national de V.P. Singh, et la victoire écrasante d'une des factions de l'Akali Dal, l'Akali Dal-M (Mann) conféra l'impression d'une consolidation ethnique sikhe, mais sans que les perspectives de la mise en oeuvre de l'accord Rajiv-Longowal ne se précisent. En février 1992, au terme de 57 mois de régime d'administration directe, des élections, caractérisées par un taux d'abstention record, (25\% de participation) portèrent au pouvoir un gouvernement congressiste sous l'égide d'un Jat-sikh, Beant Singh. A la tête des forces de police depuis 1991, Kanwar Pal Singh Gill, également Jat-sikh, considérant qu'il s'agissait d'un problème interne à régler au sein de la communauté, est parvenu en dressant les Jats contre les Jats, en l'espace de trois ans, à endiguer la vague terroriste grâce à l'élimination des chefs de file des mouvements les plus actifs. En novembre 1993, l'arrestation de Sohan Singh, principal idéologue khalistani, après l'élimination de Paramjit Singh Panjwar, leader du Khalistan Commando Force et de Daljit Singh Bittoo, chef de file de la Sikh Students Federation, marquait un pas supplémentaire dans le processus de normalisation.

Conclusion La radicalisation du communautarisme sikh, dans les années quatre-vingts, procède donc largement d'une exploitation des sentiments communautaires. La manipulation des variables ethnique et religieuse a constitué la principale stratégie des partis en rivalité dans l'arène politique locale. La responsabilité du parti du Congrès dans cette dérive politique sans précédent ne fait aujourd'hui plus de doute. L'éphémère " dictature " des fondamentalistes au Pendjab, de 1989 à 1992, aura sans doute été une leçon utile pour le jeune Etat indien, prévenu contre les dangers de promotion d'un vide politique, et d'encouragement à des mouvements communautaristes rapidement incontrôlables. Depuis 1992, l'entrée en fonction à Chandigarh d'un gouvernement congressiste, encourageant un retour aux valeurs laïques et au jeu des forces démocratiques, favorise un retour à des procédures politiques plus saines ${ }^{16}$. La lassitude des populations civiles aide naturellement l'Etat à éradiquer les réseaux terroristes qui vivaient sur le pays. L'essoufflement du mouvement séparatiste à l'étranger laisse espérer que les sentiments d'appartenance communautaire renforceront la cohésion régionale et seront moins des facteurs de désintégration nationale. Certes, des liens étroits entre le lobby sikh américain et les sénateurs du Congrès demeurent, comme l'ont révélé, à la mi-janvier 1994, les assurances du Président Clinton de se porter garant des droits du " peuple sikh ". Ces déclarations soulevèrent un tollé en Inde. Lors de la visite du Premier ministre P.V. Narasimha Rao, au printemps 1994, des formations khalistani ont organisé d'importantes manifestations ${ }^{17}$. En Inde, la crise du Pendjab aura été un avertissement 
majeur dans l'histoire politique du pays. Face aux multiples forces centrifuges, il est certain que désormais, forts de cet enseignement, les dirigeants au Centre, congressistes ou non, ne réveilleront pas sans en mesurer les déviances possibles, des communautarismes, qui constituent finalement des menaces plutôt que des facteurs de consolidation du sentiment d'appartenance dans un Etat pluri-confessionnel et multinational, dont ils s'étaient portés garants.

\section{NOTES}

1. Ce souci de garantir aux Sikhs la pérennité de leurs privilèges apparaît notamment dans les assurances données à plusieurs reprises par Jawarlal Nehru et le Mahatma Gandhi que les intérêts sikhs ne seraient pas ignorés dans de futurs engagements constitutionnels (Jawaharlal Nehru à Master Tara Singh, S. Gopal, selected works of Jawaharlal Nehru, vol. 10). Sur la question, voir également K.L. Tuteja, The "Sikhs and The Nehru Report" in Paul Wallace, Surendra Chopra, Political Dynamics and Crisis in Punjab, pp. 87-101 ; "The Sikh and The Congress, 1930-1940", pp. 102-122.

2. Pour une vision approfondie du problème, on se reportera utilement à l'étude faite par Paul Brass, Language, Religion and Politics in Northern India, Cambridge University Press, 1974. L'auteur y défend la thèse d'une Inde Etat-multinational. Il reprend ce thème de façon plus approfondie dans un ouvrage récent, Ethnicity and Nationalism, Sage, New Delhi, 1991.

3. La proportion des Sikhs sur le territoire du Pendjab a toujours été un sujet de préoccupation pour ces derniers. Constituant $56 \%$ de la population en 1966 après le redécoupage de la région, ils représentaient quelque $52 \%$ en 1981. L'importance de la population migrante en provenance des Etats du Bihar, de l'Uttar Pradesh et du Madhya Pradesh a aussi été à l'origine de la volonté d'inverser les flux migratoires afin d'asseoir une majorité sikhe, devenue au milieu des années quatre-vingts le cheval de bataille des fondamentalistes sikhs.

4. Il est à noter que les desiderata des dirigeants sikhs figuraient déjà dans les propositions constitutionnelles soumises au Comité Sapru à la veille de l'indépendance dans lequel les sikhs rappellent leur position unique, en tant que troisième communauté dans une Inde divisée entre hindous et musulmans mais au poids politique historique et économique dépassant leur importance numérique (Appendix $\mathrm{N}^{\circ}$ VII, Sikh leaders Memorandum for the Conciliation Committee, in Constitutional Proposals of The Sapru Committee, January 1946).

5. Pour une présentation rapide mais complète de l'articulation des variables ethnique et religieuse sikhes dans la conduite politique de cette minorité, on se reportera utilement à l'article de Jasmail Singh Brar, "Minorities and National Integration in India, a case study of the Sikh in the Punjab", The Sikh Review, Calcutta, March 1990. Pour une étude plus approfondie, l'ouvrage récemment publié de Rajinder Kaur, retrace dans une perspective historique le dilemme identitaire sikh (Sikh identity and National integration, New Delhi, Intellectual Publishing House, 1992). 
6. De tous les pays d'Asie du Sud, l'Inde est le seul Etat laïque par proclamation constitutionnelle. Cependant, l'idée de privatisation du religieux n'existe pas en Inde, les traditions religieuses ne reconnaissant pas les dualismes, sacré/profonde, religieux/ laïque, public/privé. Etre un Etat laïque dans le contexte des traditions religieuses et culturelles locales ne suppose pas qu'une réelle barrière sépare l'Eglise de l'Etat, à l'instar de la situation française. Dans le contexte indien, l'Etat laïque signifie que l'Etat ou le gouvernement ne discriminera pas entre les citoyens selon leur appartenance religieuse. Il créera des conditions dans lesquelles chacun sera libre de suivre sa vie religieuse et culturelle selon ses enseignements. Les fondamentalistes sikhs ont contesté le libellé de l'article 25 de la Constitution qui assimile les sikhs aux hindous. En 1984, ils ont brûlé symboliquement ces pages jugées outrageantes de la Constitution. 7. Abida Samiuddin (ed.), Punjab Crisis, Challenge and Response, New Delhi, Mittal Publications, 1985, Appendix II p. 665. Cette résolution fut référée à la Commission Sarkaria nommée par Indira Gandhi en 1983 pour réévaluer les relations Centre-Etat (Sarkaria Commission Report, Government of India Publications, 1988). Pendant la guerre du Golfe, le leader d'une des factions de l'Akali Dal, Simranjit Singh Mann, proposa de dépêcher des troupes sikhes pour appuyer les soldats américains. Dans une démarche similaire, SS. Mann adressait en novembre 1990 un mémorandum au gouvernement central appliquant les principes des relations internationales à ce qui relève des relations Centre-Etat.

8. Le Babbar Khalsa, ramification de l'Akhand Kirthani Jatha, a choisi la création de l'Etat d'Israël et le combat de Libération nationale kurde comme modèle du mouvement d'émancipation sikh.

9. En novembre 1983, un ouvrage en Punjabi, Khalsa Raj, fut diffusé en Angleterre par le Dal Khalsa, appelant l'avénement du règne de la Khalsa. Il rappelait, en outre, qu'il se proposait de réveiller les sentiments religieux des sikhs, et de les inciter à coopérer avec les autres groupes luttant pour l'indépendance, prônant la révolte armée. Des tracts, distribués en Inde le 29 mai 1983, qualifiaient la Constitution indienne de document invalide et déclaraient que le Dal Khalsa proposait de défier l'Inde devant la $\mathrm{CIJ}$ et d'intervenir auprès des Nations-Unies en faveur du mouvement "Quit Sikh Homeland".

10. Le National Council of Khalistan représente l'un des principaux artisans du séparatisme en exil. Sur l'évolution de la question khalistani et la dérive fondamentaliste, on se reportera à l'article de T.N. Madan, "Fundamentalism and the Sikh Religious Tradition", in Martin Marty and R. Scott Appleby (eds.) Fundamentalism observed, Chicago University Press, 1991, pp. 596-627. De façon significative, dans la phase d'élaboration de l'accord, avaient été exclus des négociations les plus radicaux des deux camps : Buta Singh et Darbara Singh, pour le parti du Congrès (anciens membres de l'Akali Dal), et Prakash Singh Badal, Gurbashan Singh Tohra, pour le parti Akali.

11. White Paper on Punjab Agitation pp. 105-107.

12. De nombreuses associations pour la défense des droits de l'homme s'élevèrent contre ces pratiques du gouvernement central, dénonçant les lois noires imposées à la population sikhe du Pendjab. Les années 1986 à 1991 sont dénoncées par le parti Akali comme l'avènement de l'Etat terroriste. Le 59e amendement à la Constitution, qui permit de prolonger le régime d'administration directe aux termes de l'article 356, fut aussi largement à l'origine de l'enlisement. 
13. Un comité panthique de cinq membres constitue l'organe central des quatre groupes principaux autour de la nébuleuse khalistani (Khalistan Commando Force, Khalistan Liberation Force, Babbar khalsa et All-India Sikh Students Federation). Sohan Singh, médecin dans les années cinquante et directeur des services de santé de l'Etat du Pendjab (réfugié au Pakistan), lança un appel à la sédition en 1984.

14. Sur la période 1984-1991, l'analyse de Gurharpal Singh, "The Punjab Problem in the 1990s. A post-1984 assessment", The Journal of Commonwealth and Comparative Politics, July 1991 pp. 175-189) rend le Congrès responsable de l'enlisement et de la transformation du problème pendjabi en insoluble conflit ethnique. La répression policière massive, par une police accusée de biais communautaristes au tournant des années quatre-vingt-dix, s'avéra totalement inefficace. Sur les conséquences de cette dernière, voir Ved Marwah, "Cop-ping out", Seminar n³98, december 1992.

15. Les militants s'attaquèrent aux symboles du gouvernement "hostile" de New Delhi, en prenant pour cible les représentants de la station d'émission de la All India Radio. A Chandigarh, R.K. Talib fut assassiné en décembre 1990. A Patiala, Manchanda, chef de station locale, fut décapité et sa tête envoyée au gouvernement central. (Cf. article de l'auteur, "Le Pendjab, du séparatisme à la transition démocratique", Hérodote, $\mathrm{n}^{\circ} 71$, décembre 1993).

16. Ces élections ont donné lieu à de nombreuses analyses en raison du faible taux de participation. Mais leur déroulement, sous l'égide de P.V. Narasimha Rao, qui a à son passif d'avoir été ministre de l'Intérieur au moment des pogroms anti-sikhs de Delhi, et du Gouverneur Surendra Nath, ancien directeur des forces de police de Delhi, fut une victoire en soi, le premier pas vers la restauration du processus démocratique.

17. Pour une illustration de l'influence du Pakistan dans les opérations de déstabilisation, périodiquement dénoncées par la presse indienne, on peut se reporter aux articles, "Pakistan's Involvement in Punjab Terrorism, a familiar link, another ISI Action", Frontline, 9 avril 1993 et "Peace is the first step", Pioneer, 17 juin 1992.

\section{INDEX}

Mots-clés : démocratie, Etat-nation, Nationalismes, séparatismes/ autonomismes

Index géographique : Inde 